\title{
Local Main Chain Dynamics of Phenolphthalein Polyethersulfone
}

\author{
Jianli WANG, ${ }^{*}$ Jianguo Zhang, Binyao LI, \\ and ZHILIU FeNG** \\ Changchun Institute of Applied Chemistry, Chinese Academy of Sciences, \\ Changchun, 130022, People's Republic of China
}

(Received November 18, 1993)

\begin{abstract}
Local main chain dynamics of dissolved phenolphthalein polyethersulfone (PES-C) in solution with chloroform- $d_{1}$ were examined through ${ }^{13} \mathrm{C}$ NMR relaxation measurements. Spin-lattice relaxation times and NOE (nuclear Overhauser effects) factors were measured as a function of temperature. The relaxation data were interpreted in terms of main chain segmental motion by using the damped orientational diffusion model (DAMP) and the conformation jump model (VJGM) derived by Valeur, Jarry, Geny, and Monnerie. The simulation method used is $\mathrm{N}$-SIMPLEX, which gives, in this study, a result of the object function less than $10^{-4}$. Correlation times were obtained for the main chain motion of PES-C with these models and the results indicate that the main chain of PES-C are flexible. The comparison between PES-C and 1,2-polybutadiene is proposed. The distribution of the correlation time for the main chain motion by using VJGM model is discussed. The temperature dependence of correlation times for PES-C indicating the dynamical rigidity of its chains is obtained
\end{abstract}

KEY WORDS ${ }^{13} \mathrm{C}$ NMR / phenolphthalein Polyethersulfone / Spin-lattice

Relaxation Time / Nuclear Overhauser Effect / Molecular Motion /

Correlation Time /

The macroscopic properties of polymeric materials depend on the structure, order and dynamics of the macromolecular chains, ${ }^{1}$ i.e., both the intramolecular and the intermolecular interactions contribute to the properties of polymers. Either of the contributions is important. Among a variety of physico-chemical techniques which have been applied in order to characterize these properties, ${ }^{2}$ nuclear magnetic resonance (NMR) has proven particularly useful and versatile. ${ }^{3}$

Attention can be focused on the internal motions of a single chain in a NMR solution study when the polymer solution is relatively dilute and effects of the chain-chain interactions are minimized..$^{5-8}$ Usually $10 \%$ is used in NMR relaxation mesurement and then the single chain motion can be emphasized. ${ }^{7,32}$

* To whom correspondence should be addressed.

** Formerly E.T.L. Voong and Voong Tse-lieu.
Here in this paper, we employ the solution NMR relaxation measurements to study the local main chain dynamics of phenolphthalein polyethersulfone (PES-C) to illustrate the intramolecular interactions existed in PES-C chains. It is the important basis of some further studies such as chain entanglement study, interchain charge-transfer interaction ${ }^{4}$ study in concentrated solutions or in bulk state and so on. Studies of local dynamics should provide guidance in unravelling the more complex dynamics of bulk polymers. ${ }^{5-8}$

Chain dimensions in terms of characteristic ratios as determined from rheology measurement show that the chains of polyethersulfone (PES) as well as polyetherimide (PEI), poly(2,6-dimethyl-1,4-phenylene oxide) (PPO) and polyetherketone (PEEK) are highly flexible..$^{9-14}$ 
The values of $C_{\infty}$ for these polymers are similar to those for rubber chains. How about the NMR relaxation results for both plastics and rubbers? Are they in accordance with the rheology results mentioned above or contrary to that? What's the comparison results between chains of such engineering plastics and flexible rubbers? All of these are very interesting and important questions and this paper will answer them.

Phenolphthalein polyethersulfone (PES-C) is a high performance thermoplastic resin developed in Changchun Institute of Applied Chemistry, Chinese Academy of Sciences, P. $R$. China under a Chinese patent ${ }^{15}$ which is of great interest and being paid much attention because of its technologically important properties in bulk. Usually the introduction of the methyl group or the cardo ring increases the solubility of high performance polymers such as polyethersulfone, polyimides and so on. The introduction of the phenolphthalein in the synthesis of PES-C has some favourable effects on the polymerization which is credited to the 'self-catalysis interaction' of the phenolphthalein group and the solubility of the resulted polymers are improved together with the increasing of the glass transition temperature. ${ }^{40}$ PES-C has better solubility than polyethersulfone without the cardo group. It can dissolve in more solvents than PES. This enables us to study PES-C and some rubber chains in the same solvent such as chloroform- $d_{1}$ as a good solvent for both PES-C and 1,2-polybutadiene and to compare them under the similar conditions. Up to date, there is little systematic research on the local dynamics of polyethersulfones ${ }^{16-18}$ and there is no information on that of PES-C. So the importance of our work is obvious.

\section{EXPERIMENTAL}

PES-C was supplied by Xuzhou Engineering Plastic Co., China. Its reduced viscosity in chloroform $\eta_{\mathrm{sp}} / c=0.47 \mathrm{dl} \mathrm{g}^{-1}$. For spin-lattice relaxation and NOE measurements, $10 \%(\mathrm{w} / \mathrm{v})$ solution of PES-C in chloroform- $d_{1}$ was prepared in a $5 \mathrm{~mm}$ NMR tube. The solvent was added under vacuum with the protection of highly pure Argon gas and then the sample tube was sealed.

${ }^{13} \mathrm{C}$ nuclear magnetic resonance experiments were conducted on Varian Unity 400 spectrometer operating at $100.577 \mathrm{MHz}$ for the carbon nucleus. The temperature was controlled within $0.1^{\circ} \mathrm{C}$ automatically. ${ }^{13} \mathrm{C}$ spin-lattice relaxation times were measured by the standard inversion recovery technique with a repetition time longer than $5 T_{1}$. Values of $T_{1}$ were determined with a rms error of $\pm 5 \%$ or better. ${ }^{13} \mathrm{C}$ NOE experiments were carried out by the gated decoupling with 2048 transients and delays of at least 5 times the longest $T_{1}$ were used between $90^{\circ}$ pulses. The NOE factors were measured twice and the average values were obtained.

Numerical calculations were performed by using N-SIMPLEX program, modified to include the spectral density functions of the motional models used in this study. The outstanding feature of N-SIMPLEX is that the object function is independent of the concrete form of the spectral density and it can be used in some more complex situations. The description of the program and the procedure used to obtain simulation parameters of the various models have been described elsewhere. $^{19}$

\section{INTERPRETATION}

Assuming only dipolar relaxations, the standard relationships between the ${ }^{13} \mathrm{C}$ spinlattice relaxation times $T_{1}$ 's and the spectral density, $J$, are given by ${ }^{14,15}$

$$
\begin{aligned}
1 / T_{1}= & (h / 2 \pi)^{2} \gamma_{\mathrm{C}}{ }^{2}{\gamma_{\mathrm{H}}}^{2} \sum_{i}\left[J\left(\omega_{1}\right)+3 J\left(\omega_{2}\right)\right. \\
& \left.+6 J\left(\omega_{3}\right)\right] / 20 r_{\mathrm{i}}^{6}
\end{aligned}
$$

and the nuclear Overhauser effect is given by 


$$
\begin{gathered}
N O E=1+3.976\left[-J\left(\omega_{1}\right)+6 J\left(\omega_{3}\right)\right] /\left[J\left(\omega_{1}\right)\right. \\
\left.+3 J\left(\omega_{2}\right)+6 J\left(\omega_{3}\right)\right] \\
\omega_{1}=\omega_{\mathrm{H}}-\omega_{\mathrm{C}}, \quad \omega_{2}=\omega_{\mathrm{C}}, \quad \omega_{3}=\omega_{\mathrm{H}}+\omega_{\mathrm{C}}
\end{gathered}
$$

where $\gamma_{\mathbf{C}}$ and $\gamma_{\mathbf{H}}$ are the gyromagnetic ratios for carbon and hydrogen, $\omega_{\mathrm{C}}$ and $\omega_{\mathrm{H}}$ are their resonance frequencies. $r_{\mathrm{i}}$ is the internuclear distance. The dipole-dipole relaxation of the quaternary carbons in the main chain is mainly by two methine protons of the phenyl ring. The phenyl protons in the phenolphthalein group is rather far from the main chain carbons and interaction between them can be reasonably omitted. Here the internuclar distance $r_{\mathrm{i}}$ used in the simulation is $0.215 \mathrm{~nm}$ calculated from the $\mathrm{C}-\mathrm{H}$ distance $(0.109 \mathrm{~nm})$, the $\mathrm{C}-\mathrm{C}$ bond length of the phenyl ring $(0.139 \mathrm{~nm})$ and the angle between $\mathrm{C}-\mathrm{H}$ and $\mathrm{C}-\mathrm{C}$ vectors $\left(120^{\circ}\right)$.

The spectral density in eq 1 and eq 2 is the Fourier transform of the correlation function $\Phi(t)$ which is given by ${ }^{21}$

$$
J(\omega)=\int_{-\infty}^{+\infty} \Phi(t) \mathrm{e}^{-i \omega t} \mathrm{~d} t
$$

Many models of local polymer dynamics have been proposed. We have fit the data to two models describing the local main chain motion in this paper. One is the conformational jump model for the main chain dynamics derived by Valeur, Jarry, Geny, and Monnerie (VJGM). ${ }^{22,23}$ The dipole correlation function in the VJGM model is

$$
\begin{aligned}
\Phi(t)_{\mathrm{VJGM}}= & \exp \left(-t / T_{\mathrm{o}}\right) \exp \left(-t / T_{\mathrm{d}}\right) \\
& \times \operatorname{erfc}\left[\left(t / T_{\mathrm{d}}\right)^{1 / 2}\right]
\end{aligned}
$$

where erfc is the complementary error function. $T_{\mathrm{d}}$ is supposed to represent the time associated with three-bond "Boyer-crankshaft" motions on a diamond lattice. The additional exponential decay term with $T_{0}$ was introduced to obtain the time associated with four- (or more) bond motion and diffusive motions. The spectral density obtained by Fourier transforming eq 4 and is

$$
A=T_{\mathrm{o}} T_{\mathrm{d}}\left(T_{\mathrm{o}}-T_{\mathrm{d}}\right) /\left[\left(T_{\mathrm{o}}-T_{\mathrm{d}}\right)^{2}+\omega^{2} T_{\mathrm{o}}^{2} T_{\mathrm{d}}^{2}\right]
$$

$$
\begin{aligned}
& B=\left(T_{\mathrm{o}} / 2 T_{\mathrm{d}}\right)^{1 / 2} \\
& C=\left(1+\omega^{2} T_{\mathrm{o}}^{2}\right)^{1 / 2} \\
& J(\omega)_{\mathrm{VJGM}}= A\left\{B\left[(C+1) / C^{2}\right]^{1 / 2}\right. \\
&+B\left[\omega T_{\mathrm{o}} T_{\mathrm{d}} /\left(T_{\mathrm{o}}-T_{\mathrm{d}}\right)\right] \\
&\left.\times\left[(C-1) / C^{2}\right]^{1 / 2}-1\right\}
\end{aligned}
$$

Bendler and Yaris ${ }^{24,25}$ once presented an exactly solvable model of polymer main chain dynamics called the cutoff diffusion model. Skolnick and Yaris ${ }^{23,26-29}$ developed a new model including a damping term directly in the diffusion equation while retaining the attractive feature of the model by Bendler and Yaris. It is called the damped orientational diffusion model (DAMP) and the correlation function is given by

$$
\Phi_{\text {DAMP }}(t)=\left(\mathrm{e}^{-\beta t} / 2 \omega_{\mathrm{c}}\right)(\pi / D t)^{1 / 2} \operatorname{ercf}\left[\left(D t \omega_{\mathrm{c}}^{2}\right)^{1 / 2}\right]
$$

and

$$
\delta=D \omega_{\mathrm{c}}^{2}
$$

where $D$ is the diffusion constant (jump probability), $\beta$ is the damping constant representing the cooperative conformational transition rate. $\omega_{c}$ is the short-wavelength cutoff, corresponding to the fact that there is a smallest displaceable unit in the polymer backbone. Consequently $\delta$ represents the conformational transition rate of the smallest displaceable unit in the polymer backbone. The spectral density of DAMP model is given by

$$
\begin{aligned}
J(\omega)= & \delta^{-1}\{[C(\omega) / 4 K(\omega)] \ln [A(\omega) / B(\omega)] \\
& +S(\omega) / 2 K(\omega) \\
& \left.\times \tan ^{-1}\left[2 K(\omega) S(\omega) /\left(K^{2}(\omega)-1\right)\right]\right\} \\
A(\omega)= & 1-2 K(\omega) C(\omega)+K^{2}(\omega) \\
B(\omega)= & 1+2 K(\omega) C(\omega)+K^{2}(\omega) \\
K(\omega)= & {\left[\left(\left(\omega^{2}+\beta^{2}\right) / \delta^{2}\right)^{1 / 4}\right.} \\
C(\omega)= & \left\{1 / 2\left[1-\beta /\left(\omega^{2}+\beta^{2}\right)^{1 / 2}\right]\right\}^{1 / 2} \\
S(\omega)= & \left\{1 / 2\left[1+\beta /\left(\omega^{2}+\beta^{2}\right)^{1 / 2}\right]\right\}^{1 / 2}
\end{aligned}
$$

Because of the complexity of the spectral density we use the N-SIMPLEX optimization 
method which is independent of the form of the object function to simulate the parameters of the motional models. The object function $F X$ is given by

$$
\begin{aligned}
{[1} & \left.-\left(T_{1 \text { (theo.) }} / T_{1(\text { exp. })}\right)\right]^{2} \\
& +\left[\left(1-\operatorname{NOE}_{(\text {theo. })} / \operatorname{NOE}_{(\text {exp. })}\right)\right]^{2}
\end{aligned}
$$

\section{RESULTS AND DISCUSSION}

The repeat unit structure and abbreviation of phenolphthalein polyethersulfone are shown below. The letter refers to the carbon on the very position.

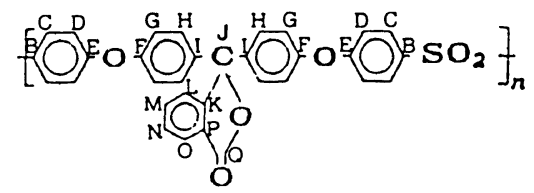

PES-C

Since we are very interested in the local main chain motion of PES-C, so the carbons in the positions, E, F, I, B, and $\mathrm{J}$ are under consideration. The chemical shift for the five carbons are 161.121, 155.509, 137.131, 136.093, and $90.643 \mathrm{ppm}$, respectively. The decay of the main chain carbons except $\mathbf{J}$ for PES-C at all temperatures followed a simple exponential dependence on delay time. The spin-lattice relaxation time $T_{1}$ is calculated from a linear least-square fit of the data in the form

$$
\ln \left(A_{\infty}-A_{\tau}\right)=\ln 2 A_{\infty}-\tau / T_{1}
$$

Figure 1 shows the temperature dependence of the ${ }^{13} \mathrm{C} T_{1}$ values for the main chain carbons of PES-C. $T_{1}$ increases with increasing temperature which indicates that the main chain motion becomes more rigorous as the temperature increases. Table I shows the average NOE values and DD\% for the main chain carbons. DD\% is the contribution of dipoledipole (DD) mechanism to the mechanisms which are available for spin-lattice relaxation. In the temperature range of $0-40^{\circ} \mathrm{C}$, the NOE values for the main chain carbons of PES-C

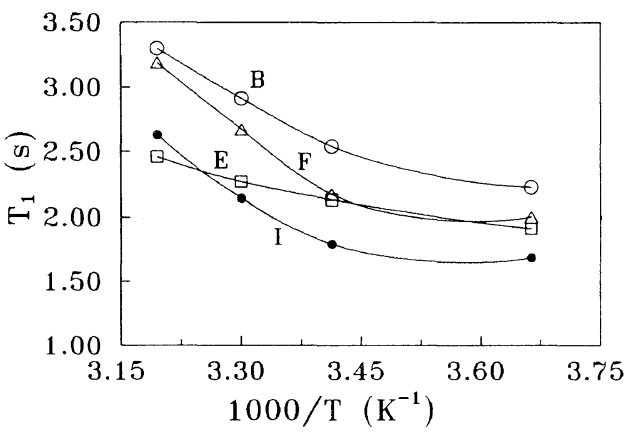

Figure 1. Temperature dependence of $T_{1}$ for the main chain carbons of PES-C in chloroform- $d_{1}$.

Table I. The average nuclear Overhauser effect and DD\% for the main chain carbons of PES-C

\begin{tabular}{lrrrr}
\hline & $\mathrm{E}^{\mathrm{a}}$ & $\mathrm{F}^{\mathrm{a}}$ & \multicolumn{1}{c}{$\mathrm{I}^{\mathrm{a}}$} & \multicolumn{1}{c}{$\mathrm{B}^{\mathrm{a}}$} \\
\hline $\mathrm{NOE}_{\mathrm{av}}$ & 1.247 & 1.208 & 1.166 & 1.309 \\
$\mathrm{DD} \%$ & $12.4 \%$ & $10.5 \%$ & $8.35 \%$ & $15.5 \%$
\end{tabular}

a E, F, I, and B represent the position in the carbons of PES-C.

do not change within the error of NOE measurement, i.e., $10-20 \%$. So the average values of NOE were summarized and used to calculated the spin-lattice relaxation times from the DD relaxation mechanism $T_{1}{ }^{\text {DD }}$ according to 21

$$
\begin{aligned}
\mathrm{DD} \% & =(\mathrm{NOE}-1) / 1.988 \\
T_{1}{ }^{\mathrm{DD}} & =T_{1} /(\mathrm{DD} \%)
\end{aligned}
$$

Table II shows the simulation results by using DAMP model. It is seen that the values of the object function are less than $10^{-4}$ which indicates the efficiency of the N-SIMPLEX optimization method. The cooperative conformational transition rate $(\beta)$ and the smallest displaceable unit conformational transition rate $(\delta)$ is in the order of $10^{9} \mathrm{~s}^{-1}$ which indicates the very fast transition rate comparable with those of flexible rubber chains as shown in Table III. ${ }^{30}$ This is in accordance with the result of the characteristic ratio. Chains of PES-C are as flexible as those of 1,2-polybutadiene. The comparison can be conducted since the two 
Table II. The simulation results for the main chain carbons of PES-C by using DAMP model

\begin{tabular}{|c|c|c|c|c|c|c|c|c|c|c|c|c|}
\hline \multirow{3}{*}{$\frac{t}{{ }^{\circ} \mathrm{C}}$} & \multicolumn{3}{|c|}{ E } & \multicolumn{3}{|c|}{$\mathrm{F}$} & \multicolumn{3}{|c|}{ I } & \multicolumn{3}{|c|}{ B } \\
\hline & $\beta$ & $\delta$ & $F X$ & $\beta$ & $\delta$ & $F X$ & $\beta$ & $\delta$ & $F X$ & $\beta$ & $\delta$ & $F X$ \\
\hline & $\begin{array}{l}\mathrm{s}^{-1} \\
\times 10^{-9}\end{array}$ & $\begin{array}{l}\mathrm{s}^{-1} \\
\times 10^{-9}\end{array}$ & $\times 10^{4}$ & $\begin{array}{c}s^{-1} \\
\times 10^{-9}\end{array}$ & $\begin{array}{c}\mathrm{s}^{-1} \\
\times 10^{-9}\end{array}$ & $\times 10^{4}$ & $\begin{array}{l}\mathrm{s}^{-1} \\
\times 10^{-9}\end{array}$ & $\begin{array}{c}s^{-1} \\
\times 10^{-9}\end{array}$ & $\times 10^{4}$ & $\begin{array}{c}s^{-1} \\
\times 10^{-9}\end{array}$ & $\begin{array}{l}\mathrm{s}^{-1} \\
\times 10^{-9}\end{array}$ & $\times 10^{4}$ \\
\hline 0 & 5.167 & 1.312 & 0.15 & 6.480 & 1.498 & 0.06 & 6.835 & 1.548 & 0.05 & 4.788 & 1.258 & 0.20 \\
\hline 20 & 5.775 & 1.398 & 0.10 & 7.034 & 1.576 & 0.05 & 7.278 & 1.610 & 0.04 & 5.497 & 1.359 & 0.12 \\
\hline 30 & 6.163 & 1.453 & 0.07 & 8.708 & 1.813 & 0.02 & 8.744 & 1.818 & 0.02 & 6.333 & 1.477 & 0.07 \\
\hline 40 & 6.703 & 1.529 & 0.06 & 10.41 & 2.133 & 0.01 & 10.74 & 2.231 & 0.01 & 7.202 & 1.600 & 0.04 \\
\hline
\end{tabular}

Table III. The simulation results for $-\mathrm{CH}_{2}-$ (VTV) of 1,2-polybutadiene by using VJGM and DAMP models.

( $1,2 \%$ refers to the percent of the 1,2-segment in 1,2-PBD)

\begin{tabular}{|c|c|c|c|c|c|c|c|}
\hline \multirow{3}{*}{$1,2 \%$} & \multicolumn{2}{|c|}{ Exptl } & \multicolumn{2}{|c|}{ VJGM } & \multicolumn{3}{|c|}{ DAMP } \\
\hline & $n T_{1}$ & & $T_{\mathrm{o}}$ & $T_{\mathrm{d}}$ & $\delta$ & $\beta$ & $\beta$ \\
\hline & $\mathrm{s}$ & & $\times 10^{10} \mathrm{~s}$ & $\times 10^{10} \mathrm{~s}$ & $\times 10^{-9} \mathrm{~s}^{-1}$ & $\times 10^{-10} \mathrm{~s}^{-1}$ & $\delta$ \\
\hline 7 & 1.14 & 2.81 & 1.521 & 1.405 & 2.703 & 1.258 & 4.65 \\
\hline 21 & 1.14 & 2.64 & 1.371 & 1.289 & 3.056 & 1.387 & 4.54 \\
\hline 45 & 0.99 & 2.97 & 1.688 & 2.625 & 2.010 & 1.006 & 5.00 \\
\hline 57 & 0.95 & 2.92 & 1.847 & 2.050 & 2.003 & 0.9939 & 4.96 \\
\hline 82 & 0.63 & 2.53 & 2.075 & 3.076 & 1.747 & 0.8246 & 4.72 \\
\hline
\end{tabular}

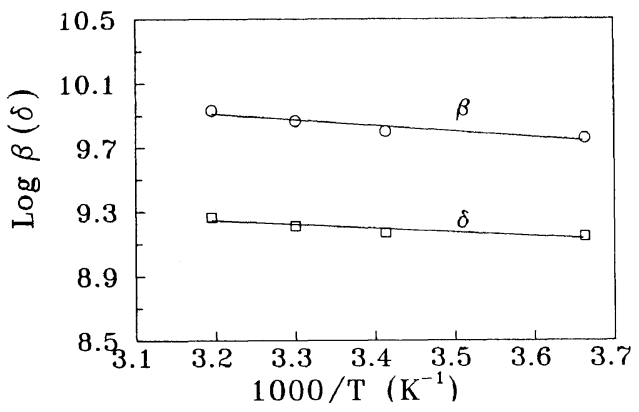

Figure 2. Temperature dependence of the chain motion parameters $\beta$ and $\delta$ of PES-C by using DAMP model.

polymers dissolve in the same solvent of the same concentration and the motional models and the similation method used are also the same. Unlike the Cole-Cole empirical model we can calculate the temperature dependence of the conformational transition rates according to ref 7. The results for PES-C are shown in Figure 2. The linear simulation equations are

$$
\begin{aligned}
& \log \beta=-0.3579 / T+11.06 \\
& \log \delta=-0.2433 / T+10.02
\end{aligned}
$$

respectively and

$$
\begin{aligned}
& E_{\mathrm{a}}(\beta)=6.85 \mathrm{~kJ} \mathrm{~mol}^{-1}, \\
& E_{\mathrm{a}}(\delta)=4.66 \mathrm{~kJ} \mathrm{~mol}^{-1}
\end{aligned}
$$

Here the lower apparent activation energy values may be somewhat due to the neglect of the temperature dependence of the NOE since the error of measurement can be $10-20 \%$. Considering that the activation energy is about $15 \mathrm{~kJ}$ mol, which indicates the dynamical rigidity of the main chain. It shows that the motional state of the main chain is relatively conserved within our temperature range, i.e., it does not change much with temperature or some other environments. The dynamical rigidity of polymer chains may be important for the intramolecular interaction. It may be due to the flexible kinks of the chain such as 
the ether bond or the sulfone group. In another research work, we use the model developed by Jones and Stockmayer to interpret the relaxation data obtained from two frequencies which are $200 \mathrm{MHz}$ and $400 \mathrm{MHz}$ and the weaker temperature dependence of the main chain motion is also obtained. ${ }^{31}$

About the relatively weaker temperature dependence of the main chain motion for polymers which contain phenyl group and the ether bond or the sulfone group or some other bonds are also shown in ref 32 and 39. The study of the unperturbed dimensions of poly(2,6-dimethyl-1,4-phenylene oxide) and poly(2,6-diphenyl-1,4-phenylene oxide) shows that the temperature dependence of the unperturbed dimension is rather weaker.. ${ }^{39}$ The unperturbed dimension of polymers has closed relationship with the chain flexibility as well as main chain motion. For the similar polymers such as polycarbonate, the apparent activation energy of the correlation time for the main chain motions obtained from NMR relaxation measurements by using another molecular motion model developed by Jones and Stockmayer is about $10-11 \mathrm{k} \mathrm{Jmol}^{-1}$ which is in accordance with our results if considering the temperature dependence of the NOE values. ${ }^{32}$ It is reasonable for some more rigid polymers such as polymethylstyrene having the strong temperature dependence of about $25 \mathrm{~kJ} \mathrm{~mol}^{-1}$. The interactions between the phenyl group and the ether bond or the sulfone group may have some correlations with the weaker temperature dependence as well as the high flexibility of these kinds of chains. However further more detailed local chain motion studies are expected to illustrate it more clearly.

In order to verify the results obtained by using DAMP model, we use the VJGM model to simulate $T_{1}{ }^{\mathrm{DD}}$ and NOE for the main chain carbons of PES-C. The simulation parameters are shown in Table IV. The values of the object function are also very small indicating the efficiency of the simulation method as that has been shown by DAMP model. The correlation times obtained for the main chain motion of PES-C are all in the order of hundreds of picosecond. The inversion of the correlation time can be comparable with the conformational transition rate obtained by using DAMP model. The correlation time decreases with the increasing temperature which means that the higher the temperature, the more rigorous the motion. Comparing the simulation parameters for PES-C by using VJGM model with those for 1,2-polybutadiene which is shown in Table III, we found that the correlation times have the same magnitude as those for 1,2-polybutadiene. The temperature dependence of $T_{\mathrm{o}}$ and $T_{\mathrm{d}}$ are shown in Figure 3. The linear simulation equations are

$$
\begin{aligned}
& -\log T_{\mathrm{o}}=-0.3845 / T+10.84 \\
& -\log T_{\mathrm{d}}=-0.2272 / T+10.42
\end{aligned}
$$

respectively and

$$
E_{\mathrm{a}}\left(T_{\mathrm{o}}\right)=7.36 \mathrm{~kJ} \mathrm{~mol}^{-1},
$$

\begin{tabular}{|c|c|c|c|c|c|c|c|c|c|c|c|c|}
\hline \multirow{3}{*}{$\frac{t}{{ }^{\circ} \mathrm{C}}$} & \multicolumn{3}{|c|}{$\mathrm{E}$} & \multicolumn{3}{|c|}{$\mathbf{F}$} & \multicolumn{3}{|c|}{ I } & \multicolumn{3}{|c|}{ B } \\
\hline & $T_{\mathrm{c}}$ & $T_{\mathrm{d}}$ & $F X$ & $T_{\mathrm{o}}$ & $T_{\mathrm{d}}$ & $F X$ & $T_{\mathrm{o}}$ & $T_{\mathrm{d}}$ & $F X$ & $T_{\mathrm{o}}$ & $T_{\mathrm{d}}$ & $F X$ \\
\hline & ps & ps & $\times 10^{-4}$ & ps & ps & $\times 10^{4}$ & ps & ps & $\times 10^{4}$ & ps & ps & $\times 10^{4}$ \\
\hline 0 & 395.5 & 267.9 & 0.33 & 310.8 & 230.2 & 0.92 & 293.7 & 222.0 & 0.63 & 439.0 & 287.0 & 2.02 \\
\hline 20 & 355.7 & 250.6 & 0.81 & 284.4 & 217.0 & 0.75 & 278.4 & 213.7 & 0.83 & 376.0 & 261.0 & 1.11 \\
\hline 30 & 327.8 & 238.4 & 1.22 & 224.2 & 191.0 & 0.31 & 224.2 & 191.0 & 0.14 & 319.3 & 234.2 & 0.78 \\
\hline 40 & 301.3 & 224.3 & 0.51 & 184.6 & 176.1 & 0.84 & 173.5 & 173.0 & 0.91 & 276.2 & 214.4 & 0.43 \\
\hline
\end{tabular}

Table IV. The simulation results for the main chain carbons by using VJGM model 


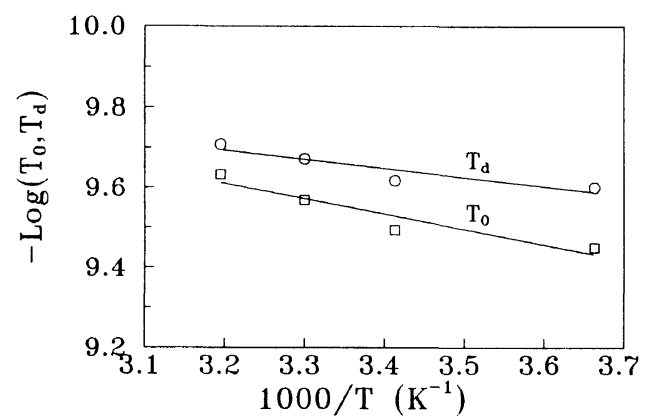

Figure 3. Temperature dependence of the chain motion parameters $T_{0}$ and $T_{\mathrm{d}}$ of PES-C by using VJGM model.

$$
E_{\mathrm{a}}\left(T_{\mathrm{d}}\right)=4.35 \mathrm{~kJ} \mathrm{~mol}^{-1} \text {. }
$$

The apparent activation energies are less than $15 \mathrm{~kJ} \mathrm{~mol}^{-1}$ which also indicates the dynamical rigidity of the main chain. These results are almost consistent with those obtained by using DAMP model which means that the two motional models are both effective to describe the main chain motion of PES-C.

As we know, the width parameter indicating the distribution of correlation time is an important factor for polymers. The assumption of isotropic reorientation with single correlation time is not suggested to characterize the backbone segmental motion. For the conformational jump model the crucial factor is the ratio $\left(T_{\mathrm{d}} / T_{\mathrm{o}}\right)$ which is dependent on the distribution of correlation time. If $\left(T_{\mathrm{d}} / T_{\mathrm{o}}\right)>10$, the model reduces effectively to the single correlation time model. ${ }^{33}$ The width parameter can be related to motional cooperativity ${ }^{34,35}$

The ratios $T_{\mathrm{d}} / T_{\mathrm{o}}$ of main chain carbons for PES-C are shown in Table V. The values of the distribution parameters listed there are subject to error because of the difficulty in measuring ${ }^{13} \mathrm{C}$ NOE values accurately and the uncertainty in bond length. In spite of these restrictions, clear trends emerge in the temperature dependence of the model parameters. They are less than unity which indicate that the distribution of correlation time is far from the single correlation time model. It is reasonable since it is expected that the correlation time for small-scale, limited local mo-
Table V. The distribution of correlation time $\left(T_{\mathrm{d}} / T_{\mathrm{o}}\right)$ for PES-C by using VJGM model

\begin{tabular}{rccccc}
\hline$t /{ }^{\circ} \mathrm{C}$ & $\mathrm{E}$ & $\mathrm{F}$ & $\mathrm{I}$ & $\mathrm{B}$ & $\left(T_{\mathrm{d}} / T_{\mathrm{o}}\right)_{\mathrm{av}}$ \\
\hline 0 & 0.68 & 0.74 & 0.76 & 0.65 & 0.71 \\
20 & 0.71 & 0.76 & 0.77 & 0.69 & 0.73 \\
30 & 0.73 & 0.85 & 0.85 & 0.73 & 0.79 \\
40 & 0.74 & 0.95 & 0.99 & 0.78 & 0.87 \\
\hline
\end{tabular}

Table VI. $\beta / \delta$ values for the main chain carbons of PES-C at different temperatures

\begin{tabular}{|c|c|c|c|c|}
\hline$\frac{t}{{ }^{\circ} \mathrm{C}}$ & $\mathrm{E}$ & $\mathrm{F}$ & I & B \\
\hline 0 & 3.94 & 4.33 & 4.42 & 3.81 \\
\hline 20 & 4.13 & 4.46 & 4.52 & 4.05 \\
\hline 30 & 4.24 & 4.80 & 4.81 & 4.29 \\
\hline 40 & 4.38 & 4.88 & 4.82 & 4.50 \\
\hline
\end{tabular}

tions will be less than that for larger scale reorietation. ${ }^{36}$ The width parameters increase systematically from $0.71-0.87$ (average value over four carbons) with increase in temperature $\left(0-40^{\circ} \mathrm{C}\right)$. The segmental motion at higher temperature is characterized by a narrower and more symmetric distribution than that at lower temperature. Thus at higher temperature the long-range cooperative motions become less important. This is in accordance with the temperature dependence which has been found for many polymers. ${ }^{37}$ However the width parameters are still significantly smaller than the values expected for single correlation time model which indicates that the distributions of correlation times are relatively wide and the long range cooperative motions are important during the experimental temperature range.

$\beta / \delta$ value obtained from DAMP model can be used to evaluate the length of the cooperative chain motion unit i.e., the length of the segment. ${ }^{30}$ The $\beta / \delta$ values for PES-C at different temperatures are shown in Table VI. The value of $\beta / \delta$ increases with the increasing temperature and the length of segmental motion unit increases. At ambient 
temperature, the inversion of the conformational transition rate of smallest displaceable unit $\left(\delta^{-1}\right)$ is no more than triple (2.8) the correlation time of the three-bond "Boyercrankshaft" motion $\left(T_{\mathrm{d}}\right)$, which means the smallest displaceable unit may be no more than six bonds and then the length of segmental motion unit will be no more than 34 bonds (calculated from $\beta / \delta=4.05$ ). $\beta / \delta$ values can be calculated from data in Table III for the content of the 1,2-segment being $82 \%$, $\beta / \delta=4.72,(1 / \delta) / T_{\mathrm{d}}=1.86$, and then the length of the cooperative motion unit is approximately 26 bonds and for the content of 1,2-segment being $21 \%$, the length is about 36 bonds. A virtual bond length for PES-C is longer than that for 1,2-polybutadiene. The average length of one bond in PES-C is approximately $0.55 \mathrm{~nm}$ (calculated from the length of one repeat unit $2.213 \mathrm{~nm}^{10}$ averaged by four bonds in it) and that in 1,2-polybutadiene is $0.154 \mathrm{~nm}(\mathrm{C}-\mathrm{C})$. So approximately the conclusion may be drawn that the cooperative motion unit length of PES- $C$ chains is only three times longer than that of 1,2-polybutadiene chains. Since these two chains have similar segment length and similar cooperative conformational transition rate, so considering the flexibility from the point of chain motion they have similar chain flexibility.

To sum up, we have studied the main chain dynamics of PES-C in chloroform through ${ }^{13} \mathrm{C}$ relaxation measurements employing two dynamic models for describing the main chain local motions by using N-SIMPLEX optimization method. It appears that both DAMP and VJGM models are effective in describing segmental motion of PES-C and N-SIMPLEX optimization method is an efficient technique for multifactor experiments. The simulation results show that PES-C chain is as flexible as 1,2-polybutadiene chain in their chloroform solution being consistent with the results of the comparison of the parameters determining the chain static flexibility such as the characteristic ratio. In solution, segmental motion of PES-C chains and that of flexible rubber chains are both very fast. We have used the model of Jones and Stockmayer to describe the segmental motion, the phenyl group rotation and the side group motion which was reported elsewhere. $^{31}$ It shows the similar result on segmental motion and the facile phenyl group with the correlation time in the order of hundreds of picosecond. These fast chain motions have connection with the properties of PES-C in bulk. For example, it is assumed that the impact strength is related to the ability of a polymer chain to undergo a rapid, reversible conformational transition without bond rupture. ${ }^{16}$ The fast chain motion of PES-C may be the source of its substantial impact strength in the glassy state. Now further investigations and the research on the intermolecular interactions are in progress.

\section{REFERENCES}

1. I. M. Ward, "Mechanical Properties of Solid Polymers," Wiley, New York, N. Y., 1971.

2. R. T. Bailey, A. M. North, and R. A. Pethrick, "Molecular Motions in High Polymers," Clarendon, Oxford, 1981.

3. F. A. Boyer, Nuclear Magnetic Resonance Spectroscopy," 2nd edition, Academic, New York, N. Y., 1987.

4. Zhenhua Sun, Haiying Li, Yugang Zhuang, Mengxian Ding, and Zhiliu Feng, Polym. Bull., 26, 557 (1991).

5. J. J. Connolly, E. Gordon, and A. A. Jones, Macromolecules, 17, 722 (1984).

6. G. C. Levy and D. H. Wang, Macromolecules, 19, 1013 (1986).

7. D. J. Gisser, S. Glowinkowski, and M. D. Ediger, Macromolecules, 24, 4270 (1991).

8. M. E. Nedea, R. H. Marchessault, and P. Dais, Polymer, 33183 (1992).

9. O. H. Schonherr, A. Schneller, A. M. Seifert, M. Soliman, and J. H. Wendorff, Makromol. Chem., 193, 1955 (1992).

10. S. A. Pavlova, G. I. Timofeeva, I. A. Ronova, and L. A. Pancratova, J. Polym. Sci., Polym. Phys. Ed., 18, 1 (1980).

11. V. A. Zubkov, T. M. Birshtein, and H.C. Mulefskaya, Vysokomol. Soedin., Ser. A, 16, 2438 (1974).

12. V. A. Zubkov et al., Vysokomol. Soedin., Ser. A, 17, 1955 (1975). 
13. T. M. Birshtein, Vysokomol. Soedin., Ser. A, 19, 54 (1977).

14. T. M. Birshtein, Vysokomol. Soedin., Ser. A, 21, 1990 (1979).

15. K. J. Liu, H. C. Zhang, and T. L. Chen, Chinese patent, No. 85101721.5 (1987).

16. A. E. Tonelli, Macromolecules, 5, 558 (1972).

17. W. H. Stockmayer, A. A. Jones, and T. L. Treadwell, Macromolecules, 10, 762 (1977).

18. A. E. Tonelli, Macromolecules, 6, 503 (1973).

19. D. H. Wang, G. X. Wang, and S. Z. Mao, Chinese J. Magn. Reson., 7, 245 (1990).

20. D. Doddrell, V. Glushko, and A. Allerhand, J. Chem. Phys., 56, 3683 (1972).

21. Z. W. Qiu and F. Q. Pei, "Nuclear Magnetic Resonance," Science, Beijing, 1989.

22. B. Valeur, J. Jarry, P. Geny, and L. Monnerie, $J$. Polym. Sci., Polym. Phys. Ed., 13, 667, 675, and 2251 (1975).

23. J. Skolnick and R. Yaris, Macromolecules, 15, 1041 1046 (1982).

24. J. T. Bendler and R. Yaris, Macromolecules, 11, 650 (1978).

25. Errata, F. Heatley, and J. T. Bendler, Polymer, 20, 1578 (1979).

26. H. Yamakawa, J. Skolnick, and R. Yaris, Macromolecules, 16, 491, 492 (1983).
27. J. Skolnick and R. Yaris, Macromolecules, 16, 266 (1983).

28. B. B. Pant, J. Skolnick, and R. Yaris, Macromolecules, 18, 253 (1985).

29. J. Skolnick and R. Yaris, Macromolecules, 18, 1635 (1985).

30. S. R. Ni, H. F. Zhang, and J. J. Liu, Acta. Polym. Sinica, 5, 580 (1990).

31. J. L. Wang, J. G. Zhang, B. Y. Li, and Z. L. Feng, submitted to Polymer.

32. J. F. O'Gara, S. G. Desjardins, and A. A. Jones, Macromolecules, 14, 64 (1981).

33. Y. Inoue, Y. Kawamura, and T. Konno, Polymer, 23, 817 (1982).

34. J. Schaefer, Macromolecules, 6, 882 (1973).

35. J. Schaefer, Topics in Carbon-13 NMR Spectroscopy, Vol. 1, G. C. Levy, Ed., Wiley-Interscience, New York, N. Y., 1975, Chapter 4.

36. F. Heatley and A. Begum, Polymer, 17, 399 (1976).

37. F. Heatley, Prog. NMR Spectrosc., 13, 47 (1979).

38. T. Akihiro and C. Clande, Macromolecules, 12, 3, 429 (1979).

39. P. J. Akers, G. Allers, and M. J. Bethell, Polymer, 9, 575 (1968).

40. X. M. Jin and K. J. Liu, Acta Polymerica Sinica, 3, 237 (1988). 